\title{
Age and Sex Differences in Sputum Smear Microscopy Results for Acid Fast Bacilli in a Tertiary Care Centre, South India
}

\author{
Palanivel Chinnakali, ${ }^{1}$ Kalaiselvi Selvaraj, ${ }^{1}$ Pruthu Thekkur, ${ }^{1}$ Gomathi Ramasamy,, \\ Mahalakshmy Thulasingam, ${ }^{1}$ and Kavita Vasudevan ${ }^{2}$ \\ ${ }^{1}$ Department of Preventive and Social Medicine, Jawaharlal Institute of Postgraduate Medical Education \& Research (JIPMER), \\ Dhanvantri Nagar, Puducherry 605006, India \\ ${ }^{2}$ Indira Gandhi Medical College \& Research Institute, Puducherry 605010, India
}

Correspondence should be addressed to Palanivel Chinnakali; palaniccm@gmail.com

Received 30 June 2014; Revised 23 September 2014; Accepted 6 October 2014; Published 9 November 2014

Academic Editor: María Sasiain

Copyright (C) 2014 Palanivel Chinnakali et al. This is an open access article distributed under the Creative Commons Attribution License, which permits unrestricted use, distribution, and reproduction in any medium, provided the original work is properly cited.

Background and Objectives. Low counts are more difficult to find in microscopic sputum examination and thus are more likely to be missed. In this study, we aimed to estimate the proportion of low-count grading and assessing any age and gender differences in sputum smear grading in a low HIV prevalence setting. Materials and Methods. From the tuberculosis laboratory register information on sputum positivity including the grading of smears, age and gender were extracted for January 2011-December 2011. Smears were examined using Ziehl-Neelsen technique and graded as per the Program Guidelines. Positive smears were classified into low grade positive smears (scanty and $1+$ ) and high grade positive smears ( $2+$ and $3+$ ). Differences in grading of smear based on gender and age were analysed using chi square test. Results. Of 9000 smears examined, 8210 (91.2\%) were collected as diagnostic smears from tuberculosis suspects. Low grade positivity was 37.5\% among diagnostic smears and $69.6 \%$ among follow-up smears. Sputum smears from female examinees had higher proportions of low grade positive smears $(P=0.003)$. Stratification of age and sex within TB suspects had clearly demonstrated the observance of higher low grade positivity among female TB suspects at extremes of age.

\section{Introduction}

Sputum smear microscopy remains the mainstay in the diagnosis of pulmonary tuberculosis in many developing countries including India. Though newer techniques like real time cartridge based nucleic acid amplification test (CBNAAT) with higher sensitivity and specificity have been introduced in fewer centres in India; good quality sputum microscopy remains the backbone in the diagnosis of pulmonary tuberculosis under Revised National Tuberculosis Control Program (RNTCP). Sensitivity (probability of finding the acid fast bacilli if present) of the sputum smear microscopy depends on many factors like quality of sputum obtained from patients, concentration of bacilli in the sputum, skill of the laboratory personnel in staining procedure, and duration between sputum sample collection and smear examination [1]. Grading of sputum smear microscopy in a patient population may differ due to difference in patient characteristics like the severity of disease, HIV status, and previous or current medication $[2,3]$.

Grading of sputum smear can be used as crude indicator of transmissibility of tuberculosis from patients to household and other contacts $[4,5]$. It also predicts the speed of bacteriologic conversion once patients were initiated on chemotherapy [4-6]. Low counts are more difficult to find in microscopic sputum examination compared to higher counts (grade) and thus are more likely to be missed [7]. The frequency of low-count grading results compared with high count grading has been considered as an indicator of the quality of sputum smear microscopy [8]. Hence periodic analysis of relative frequency of low count will improve the efficiency of sputum examination. Also, age and gender differences in grading of smears were reported in high HIV prevalence settings [9]. This has not been studied yet in 
low HIV prevalent settings of India. In this study, we aim to estimate the relative frequency of low-count grading and assessing any age and gender differences in sputum smear grading in a low HIV prevalence setting.

\section{Materials and Methods}

2.1. Study Design and Setting. A record based retrospective study was conducted using the RNTCP laboratory register maintained at Designated Microscopy Centre (DMC) of a tertiary care hospital in Puducherry, South India. In Puducherry, the prevalence of HIV for the year 2011 was 0.15 as compared to the national estimate of $0.27 \%$ [10]. In 2013-2014, among the total patients under RNTCP, 3\% were found to be HIV +ve [11]. Diagnosis of sputum smear positive tuberculosis was done as per RNTCP guidelines. All tuberculosis suspects (those who have cough for 2 weeks or more duration) were referred to DMC for sputum smear examination. Spot sputum sample was collected on the same day and the patients were advised to collect early morning sample on the next day. Laboratory technician from DMC will give container for collection of early morning sputum with specific instructions on proper collection of early morning sputum [12]. On an average, DMC examined about 30-40 sputum smear samples per day including the sputum samples of patients (followup sputum examination) who were on DOTS. Smears were prepared by Ziehl-Neelsen technique and examined under oil immersion using conventional microscope. Grading of smears was mentioned in the laboratory register as per the RNTCP guidelines. Laboratory technicians were appointed in DMCs after received training under "Revised National Tuberculosis Control Programme." Smears examined in DMCs were subjected to quality control mechanisms such as internal quality control, external quality assurance (on-site evaluation and random blinded rechecking) [8]. Though fluorescent microscopy was introduced in few medical colleges in India, during the study period, it was not rolled out in this DMC.

2.2. Study Population. Patients having cough more than two weeks duration (TB suspects) referred to DMC and patient already under DOTS therapy who came for followup examination during January 2011-December 2011 were considered to be eligible for this study. Smears obtained from examinees were considered for analysis instead of examinees. Smears which had missing data on grading were excluded. Individuals who gave both early morning and spot sputum samples were considered for further analysis.

2.3. Study Variables and Data Extraction. Information on age, sex, address, tuberculosis laboratory serial number, type of sample (spot/early morning), smear obtained for diagnosis or follow-up, and grading of smears (negative, scanty, $1+, 2+$, $3+$ ) was routinely entered in tuberculosis laboratory register. For the study purpose, we extracted information on age, sex, type of sputum (spot/early morning), grading of smears, reason for examination (diagnosis or follow-up) which was extracted in a pro forma. To simplify the grading, smears were again classified as low grade positive (scanty and 1+ smears) and high grade positive $(2+$ and $3+)$ smears. The study protocol was reviewed and approved by the Institute Ethics Committee of the tertiary care hospital in Puducherry.

2.4. Data Management. Data were entered in EpiData version 3.1 and analyzed using STATA 11.2 (StataCorp, Texas USA). Grading of smears was presented as proportions. Results were categorized according to age, sex, and diagnostic smear/follow-up smears. Chi square test was performed to assess the difference in proportions among categorical variables like sex and age groups. Confounders and effect modifiers were identified from Mantel-Haenszel odds ratio measures. Confounders were adjusted by multivariate analysis. Low grade smears (yes)/(no) were entered as dependent variable; gender (male, female), type of sample (TB suspects/follow-up), and age group (15-34 yrs, 35-54 yrs, 55-98 yrs) were entered as independent variables by enter method. Results of the adjusted analysis were presented as OR $_{\text {adj }}$ with 95\% CIs.

\section{Results}

A total of 4634 individuals had been examined for acid fast bacilli at the DMC during the reference period. Of 4614, 3\% (134) submitted only one sample and hence excluded from analysis. Of 4500 patients, $63 \%$ were males and $37 \%$ were females. Median age of male examinees was 47 (IQR 35$59 \mathrm{yrs})$ and of female examinees was 41 (28-55 yrs). Male examinees were significantly elder than female examinees $(P=0.0001)$. Out of the 4500 examinees, $395(8.8 \%)$ were on antituberculosis treatment and come for follow-up sputum examination. Of total 9000 smears examined during the reference period, $8210(91.2 \%)$ of smears were collected for diagnosis of tuberculosis among TB suspects and 790 (8.8\%) were collected as follow-up smears. Smear positivity was $14 \%$ among diagnostic smears and $14.6 \%$ among followup smears. Low grade positivity was $37.5 \%$ among diagnostic smears and $69.6 \%$ among smears on follow-up examination (Table 1).

Among the TB suspects, proportion of scanty smears and $1+$ positive smear among female examinees were higher compared with males and it was not statistically significant (data not shown). When the smears were classified as low grade or high grade, smears from female examinees had higher proportions of low grade positive smears compared to smears from male examinees $(P=0.003)$ (Table 2$)$. Similarly, low grade positive smears were more frequently seen in the extremes of age groups (15-34 yrs and 55-98 yrs) compared to middle age (25-44 yrs) (Table 2 ).

Initial screening of stratum specific estimates for all potential variables through Mantel-Haenszel odds ratio estimations did not show any statistically significant effect modifier (stratum specific OR remained similar across stratum and test of homogeneity of odds ratio for all potential variables had $P>0.05$ ). Smears obtained from female examinees, age $>55 \mathrm{yrs}$, and smears obtained during follow-up phase of illnesses had significantly higher tendency to have low grade positive smears compared to male examinees, age $<55 \mathrm{yrs}$, and smears obtained from tuberculosis suspects, respectively (Table 2). 
TABLE 1: Distribution of age, gender, and grading of smears among examinees $(N=9000)$.

\begin{tabular}{|c|c|c|}
\hline Characteristics & $\begin{array}{c}\text { New (diagnostic } \\
\text { smears) } \\
n=8210\end{array}$ & $\begin{array}{c}\text { Follow-up smears } \\
n=790\end{array}$ \\
\hline \multicolumn{3}{|l|}{ Gender } \\
\hline Male & $5194(63.3)$ & $588(74.4)$ \\
\hline Female & $3016(36.7)$ & $202(25.6)$ \\
\hline \multicolumn{3}{|l|}{ Age groups (years) } \\
\hline $14-24$ & $992(12.1)$ & $137(17.3)$ \\
\hline $25-34$ & $1258(15.3)$ & $70(8.9)$ \\
\hline $35-44$ & $1550(18.9)$ & $248(31.4)$ \\
\hline $45-54$ & $1640(20.0)$ & $196(24.8)$ \\
\hline $55-64$ & $1484(18.1)$ & $68(8.6)$ \\
\hline 65 and above & $1210(14.7)$ & $64(8.1)$ \\
\hline Age not recorded & $76(0.9)$ & $7(0.9)$ \\
\hline \multicolumn{3}{|l|}{ Smear status } \\
\hline Smear positive & $1147(14.0)$ & $115(14.6)$ \\
\hline Smear negative & $7063(86.0)$ & $675(85.4)$ \\
\hline \multicolumn{3}{|l|}{ Smear positivity } \\
\hline Scanty & $131(11.4)$ & $34(29.6)$ \\
\hline $1+$ & $299(26.1)$ & $46(40.0)$ \\
\hline $2+$ & $334(29.1)$ & $23(20.0)$ \\
\hline $3+$ & $383(33.4)$ & $12(10.4)$ \\
\hline \multicolumn{3}{|l|}{ Grading of smears } \\
\hline Low grade positivity & $430(37.5)$ & $80(69.6)$ \\
\hline High grade positivity & $717(62.5)$ & $35(30.4)$ \\
\hline
\end{tabular}

TABLE 2: Multivariate analysis on low grade positive smears with demographic and illness related factors.

\begin{tabular}{|c|c|c|}
\hline Factors & $\begin{array}{l}\text { Unadjusted odds } \\
\text { ratio }(95 \% \mathrm{CI})\end{array}$ & $\begin{array}{l}\text { Adjusted odds } \\
\text { ratio }(95 \% \mathrm{CI})\end{array}$ \\
\hline \multicolumn{3}{|l|}{ Gender } \\
\hline Male & 1.00 & 1.00 \\
\hline Female & 1.57 (1.13 to 2.18$)$ & $1.69(1.19$ to 2.39$)$ \\
\hline \multicolumn{3}{|l|}{ Samples obtained from } \\
\hline Tuberculosis suspects & 1.00 & 1.00 \\
\hline Follow-up & $4.02(2.63$ to 6.13$)$ & $4.52(2.95$ to 6.95$)$ \\
\hline \multicolumn{3}{|l|}{ Age group } \\
\hline $15-34$ yrs & 1.00 & 1.00 \\
\hline $35-54$ yrs & $0.80(0.59$ to 1.09$)$ & $0.86(0.62$ to 1.20$)$ \\
\hline $55-98$ yrs & $1.31(0.93$ to 1.85$)$ & $1.55(1.07$ to 2.23$)$ \\
\hline
\end{tabular}

Figure 1 shows proportions of low grade positive smears in age groups categorized by gender among individuals who attended DMC for sputum examination. All blue lines depict point estimate and 95\% CI of low grade positive smears among males and red line represents estimates among females.

Stratification of age and sex within TB suspects and follow-up cases clearly demonstrated the observance of

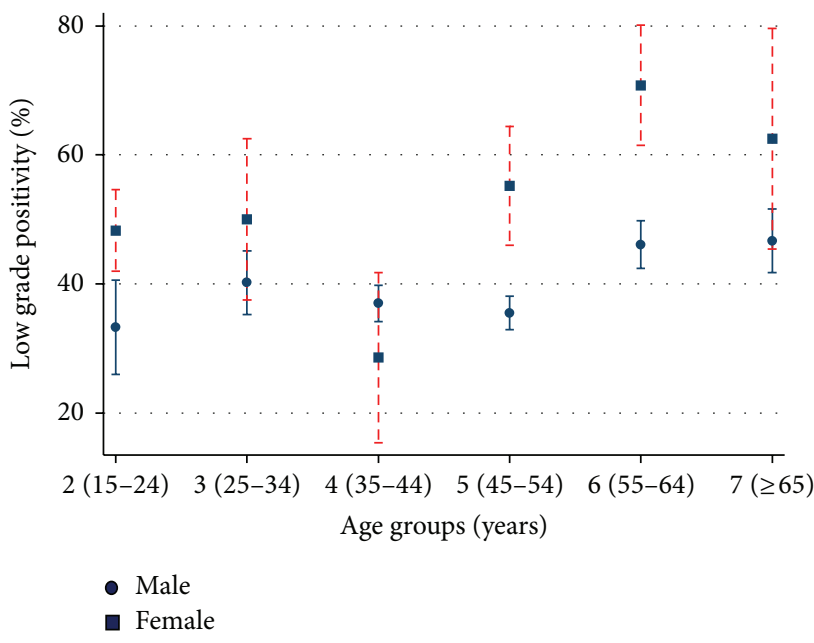

FIGURE 1: Distribution of low grade positivity based on age and gender.

higher low grade positivity among female examinees of extremes of age (Figure 1). This included all types of smears regardless of whether the examinee is a tuberculosis suspects or follow-up tuberculosis patients.

\section{Discussion}

This study showed a higher low positivity (scanty and 1+ smears) rate among females (47.8\%) and age groups 1524 yrs (40\%) and above 55 yrs (43.6-47.5\%). This difference based on gender and age group was significant for smears obtained for diagnosis among TB suspects but not among the follow-up smears. Exact reasons for this pattern are not known. This could be due to interaction of various biological, epidemiological, and social factors. Estradiol sexual hormone acts as an enhancer of cell mediated immunity response through macrophage activation by IFN-gamma thereby the lower susceptibility for tuberculosis against females [13]. Evidences have shown that males are more prone to get severe form of tuberculosis like cavitary lesions and so forth $[14,15]$. Due to fear of social stigma and the impact of disease on marital conflict female tends to overlook their disease status $[16,17]$. Also, previous studies had reported that quality of sputum given by female examinees as poor [18]. Data from the National AIDS program also showed higher prevalence of HIV among females [10]. Individuals who had HIV tend to have low grade positive smears compared to HIV negative individuals [3]. These interrelated factors ultimately could have led to more prevalence of low grade positive smears among female examinees [19-21]. Though DMC tests routinely HIV status among tuberculosis cases, the testing coverage was very low at the time of the study. Also, this study did not capture information on manifestations of disease and quality of sputum provided. Sex based difference is not only seen among low grade positive smears, actual smear positivity also grossly varied by sex. Males had significantly higher sputum positivity rate (18.5\% versus $5.2 \%$ ) during the phase of diagnosis as well as during follow-up period (19.0\% versus 
$5.3 \%)$ compared to females. TB suspects belonging to 1524 yrs of age and 55 yrs or more had significantly higher low grade positivity rate compared to other age groups. This could be caused by nonextensive lesions among extremes of age group [22]. Similar observations of high low grade positivity among females and extreme age groups were also reported in studies from few African countries [9, 23].

Grading of sputum smears had various utility such as indicator of efficiency of transmission, cure rate, and performance of sputum smear examination. Moreover, considering the low grade positivity among certain age groups the World Health Organization changed the criteria for diagnosis for $\geq 10 / \mathrm{HPF}$ to $>1 / \mathrm{HPF}$ acid fast bacilli per smear [7]. This change in diagnostic criteria helped to find out majority of missed cases especially among females. Around $10 \%$ of diagnostic smears and $50 \%$ of follow-up smears were found to be scanty smears [24]. In this study, the proportion of scanty smears was found to be around $11.4 \%$ and $29.6 \%$ among diagnostic and follow-up smears, respectively.

This study was carried out in single DMC and hence possibility of interrater variability is ruled out. Since this DMC is functioning under RNTCP, all smears were subjected to quality control mechanisms such as internal quality control, on-site evaluation, and random blinded rechecking.

Study findings should be interpreted with caution considering these following limitations. From all individuals two smears were collected. Within the same individual one smear could have been reported as scanty and the other could have been reported as $2+$. However, the disparity of smear grading within the individual was less than $2 \%$. We did not further categorize low positivity rate based on time when the sputum was collected (spot or early morning sample), since this DMC is one of the medical colleges based referral health facility where majority of the attendees were referred by medical officers from peripheral health institutions or from private. Hence, the probability of person attending at the late stage of illness is more in these kinds of DMC possible [25]. Person attending at late stage of illness is more likely to have high grade smears. In peripheral health institutes major proportions of the people will attend at early stage of illness compared to those who are attending the tertiary health institutes. Hence, at any other facility the proportions of low grade positivity will not be less than $35 \%$.

Studies have proven that simple instructions on how to collect quality sputum can substantially reduce sex disparities on sputum positivity rate [18]. Also, studies had showed that introducing fluorescent microscopy will also grossly reduce this sex disparity in grading of smears. Nevertheless, in developing countries, facilities like fluorescent microscopy, geneXpert, are beyond the reach of below district level health facilities. Hence, the programme on "Revised National Tuberculosis Control Programme" still relies on sputum smear microscopy to determine the disease status and treatment failure of an individual provided they are subjected to quality control mechanisms.

The recent change in diagnostic guidelines of two smears examination and two weeks duration of cough does not seem to have any influence on amount of time spent in examining the smears. There is no sex or age based difference in low grade positivity of smears during the follow-up period. This may be one of the reasons for nondifferential cure rates in males and females.

Though the difference in low grade positivity rate could be explained by many factors its practical implication would be towards improved diagnosis among vulnerable groups such as females and extremes of age groups. Low grade positive smears (scanty/1+) are high likely to be missed compared to high grade positive smears. Hence, highly prevalent low grade positivity smears among females may indicate that there could be a more number of missed cases among females and TB suspects belonging to either 15-24 yrs of age or aged 55 yrs or more.

\section{Conflict of Interests}

The authors declare that there is no conflict of interests regarding the publication of this paper.

\section{References}

[1] M. Sakundarno, N. Nurjazuli, S. P. Jati et al., "Insufficient quality of sputum submitted for tuberculosis diagnosis and associated factors, in Klaten district, Indonesia," BMC Pulmonary Medicine, vol. 9, article 16, 2009.

[2] A. M. Elliott, K. Namaambo, B. W. Allen et al., "Negative sputum smear results in HIV-positive patients with pulmonary tuberculosis in Lusaka, Zambia," Tubercle and Lung Disease, vol. 74, no. 3, pp. 191-194, 1993.

[3] M. C. Raviglione, J. P. Narain, and A. Kochi, "HIV-associated tuberculosis in developing countries: clinical features, diagnosis, and treatment," Bulletin of the World Health Organization, vol. 70, no. 4, pp. 515-526, 1992.

[4] K. K. Liippo, K. Kulmala, and E. O. J. Tala, "Focusing tuberculosis contact tracing by smear grading of index cases," The American Review of Respiratory Disease, vol. 148, no. 1, pp. 235236, 1993.

[5] S. Tiwari, A. Kumar, and S. K. Kapoor, "Relationship between sputum smear grading and smear conversion rate and treatment outcome in the patients of pulmonary tuberculosis undergoing dots-a prospective cohort study," Indian Journal of Tuberculosis, vol. 59, no. 3, pp. 135-140, 2012.

[6] P. G. Gopi, V. Chandrasekaran, R. Subramani et al., "Association of conversion \& cure with initial smear grading among new smear positive pulmonary tuberculosis patients treated with Category I regimen," Indian Journal of Medical Research, vol. 123, no. 6, pp. 807-814, 2006.

[7] A. Ramsay, M. Bonnet, L. Gagnidze, W. Githui, F. Varaine, and P. J. Guérin, "Sputum, sex and scanty smears: new case definition may reduce sex disparities in smear-positive tuberculosis," International Journal of Tuberculosis and Lung Disease, vol. 13, no. 5, pp. 613-619, 2009.

[8] "RNTCP EQA protocol re-drafted 09.doc," RNTCP Lab Network Guidelines, 2009, http://www.tbcindia.nic.in/pdfs/ RNTCP\%20Lab\%20Network\%20Guidelines.pdf.

[9] H. L. Rieder, J. M. Lauritsen, N. Naranbat, A. Katamba, D. Laticevschi, and B. Mabaera, "Quantitative differences in sputum smear microscopy results for acid-fast bacilli by age and sex in four countries," International Journal of Tuberculosis and Lung Disease, vol. 13, no. 11, pp. 1393-1398, 2009. 
[10] India HIV Estimates Technical Report, 2012, http://www.naco. gov.in/upload/Surveillance/Reports $\% 20 \& \% \% 20$ Publication/ Technical\% 20Report\% 20 - \% 20India \% 20 HIV \% 20Estimates $\% 202012$.pdf.

[11] "Annual status report," TB India 2014, Revised National TB Control Programme, Government of India, 2014, http://www. tbcindia.nic.in/pdfs/TB\%20INDIA\%202014.pdf.

[12] Revised National Tuberculosis Control Programme (RNTCP), Module for Laboratory Technicians, 2005, http://tbcindia.nic. in/pdfs/Module\%20for\%20Laboratory\%20Technician.pdf.

[13] O. Neyrolles and L. Quintana-Murci, "Sexual inequality in tuberculosis," PLoS Medicine, vol. 6, no. 12, Article ID e1000199, 2009.

[14] A. Thorson, N. H. Long, and L. O. Larsson, "Chest X-ray findings in relation to gender and symptoms: a study of patients with smear positive tuberculosis in Vietnam," Scandinavian Journal of Infectious Diseases, vol. 39, no. 1, pp. 33-37, 2007.

[15] E. Johansson, N. H. Long, V. K. Diwan, and A. Winkvist, "Gender and tuberculosis control: perspectives on health seeking behaviour among men and women in Vietnam," Health Policy, vol. 52, no. 1, pp. 33-51, 2000.

[16] D. Somma, B. E. Thomas, F. Karim et al., "Gender and sociocultural determinants of TB-related stigma in Bangladesh, India, Malawi and Colombia," International Journal of Tuberculosis and Lung Disease, vol. 12, no. 7, pp. 856-866, 2008.

[17] G. D. Gosoniu, S. Ganapathy, J. Kemp et al., "Gender and sociocultural determinants of delay to diagnosis of TB in Bangladesh, India and Malawi," International Journal of Tuberculosis and Lung Disease, vol. 12, no. 7, pp. 848-855, 2008.

[18] M. S. Khan, O. Dar, C. Sismanidis, K. Shah, and P. GodfreyFaussett, "Improvement of tuberculosis case detection and reduction of discrepancies between men and women by simple sputum-submission instructions: a pragmatic randomised controlled trial," The Lancet, vol. 369, no. 9577, pp. 1955-1960, 2007.

[19] R. Balasubramanian, R. Garg, T. Santha et al., "Gender disparities in tuberculosis: report from a rural DOTS programme in South India," International Journal of Tuberculosis and Lung Disease, vol. 8, no. 3, pp. 323-332, 2004.

[20] V. Begum, P. De Colombani, S. Das Gupta et al., “Tuberculosis and patient gender in Bangladesh: sex differences in diagnosis and treatment outcome," International Journal of Tuberculosis and Lung Disease, vol. 5, no. 7, pp. 604-610, 2001.

[21] F. Karim, F. Ahmed, I. Begum, E. Johansson, and V. K. Diwan, "Female-male differences at various clinical steps of tuberculosis management in rural Bangladesh," The International Journal of Tuberculosis and Lung Disease, vol. 12, no. 11, pp. 1336-1339, 2008.

[22] C. Pérez-Guzmán, M. H. Vargas, A. Torres-Cruz, and H. Villarreal-Velarde, "Does aging modify pulmonary tuberculosis?: A meta-analytical review," Chest, vol. 116, no. 4, pp. 961-967, 1999.

[23] L. Lawson, M. A. Yassin, A. N. Onuoha et al., "Yield of smear microscopy and radiological findings of male and female patients with tuberculosis in abuja, Nigeria," Tuberculosis Research and Treatment, vol. 2010, Article ID 241659, 5 pages, 2010.

[24] A. van Deun, A. Hamid Salim, E. Cooreman et al., "Scanty AFB smears: what's in a name?" International Journal of Tuberculosis and Lung Disease, vol. 8, no. 7, pp. 816-823, 2004.

[25] L. Otero, R. Ugaz, G. Dieltiens et al., "Duration of cough, TB suspects' characteristics and service factors determine the yield of smear microscopy," Tropical Medicine and International Health, vol. 15, no. 12, pp. 1475-1480, 2010. 


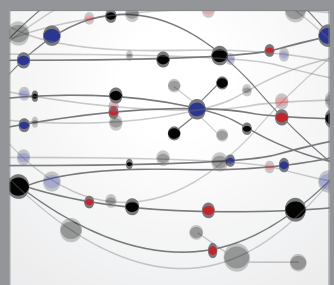

The Scientific World Journal
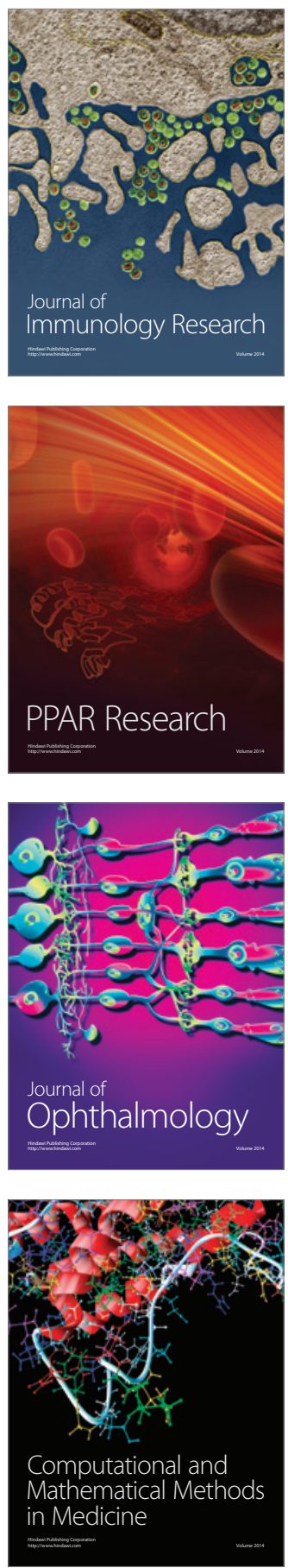

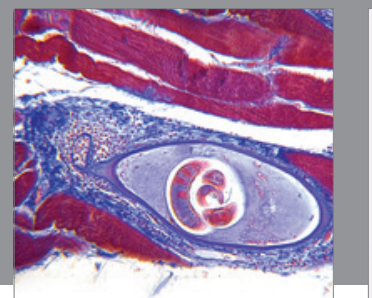

Gastroenterology

Research and Practice
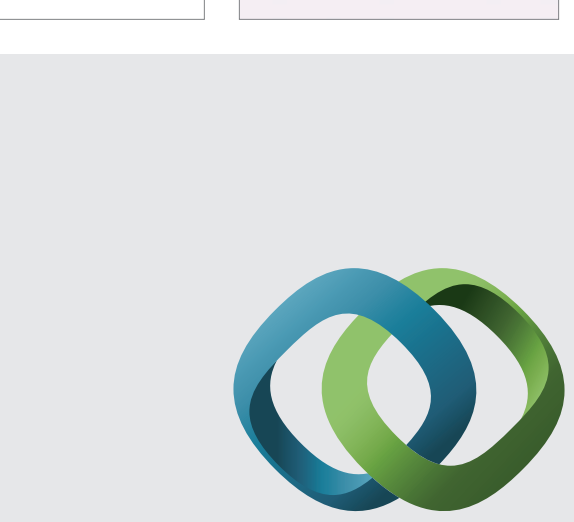

\section{Hindawi}

Submit your manuscripts at

http://www.hindawi.com
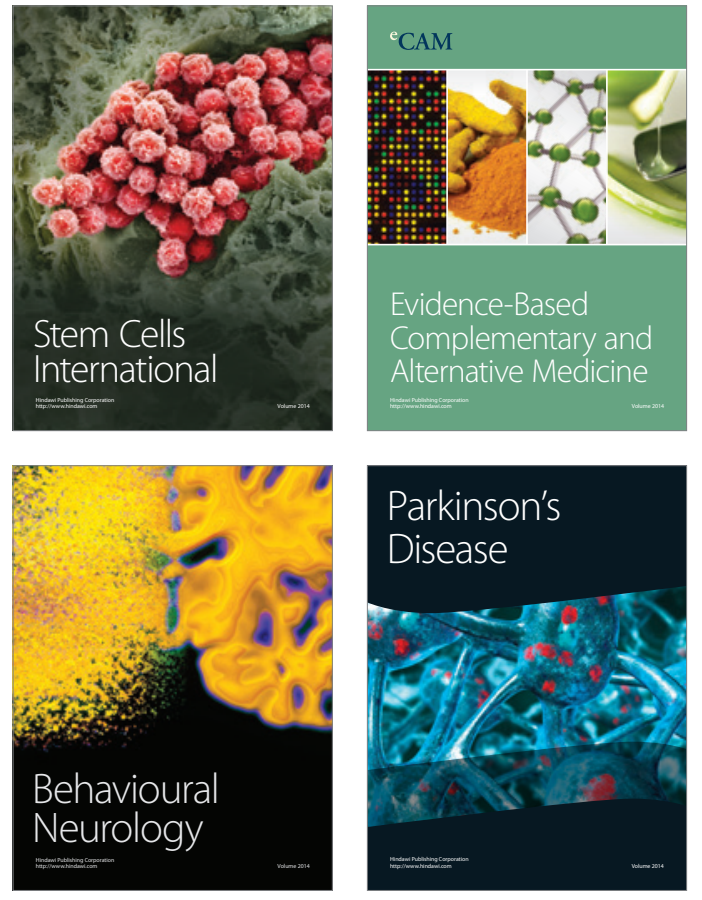
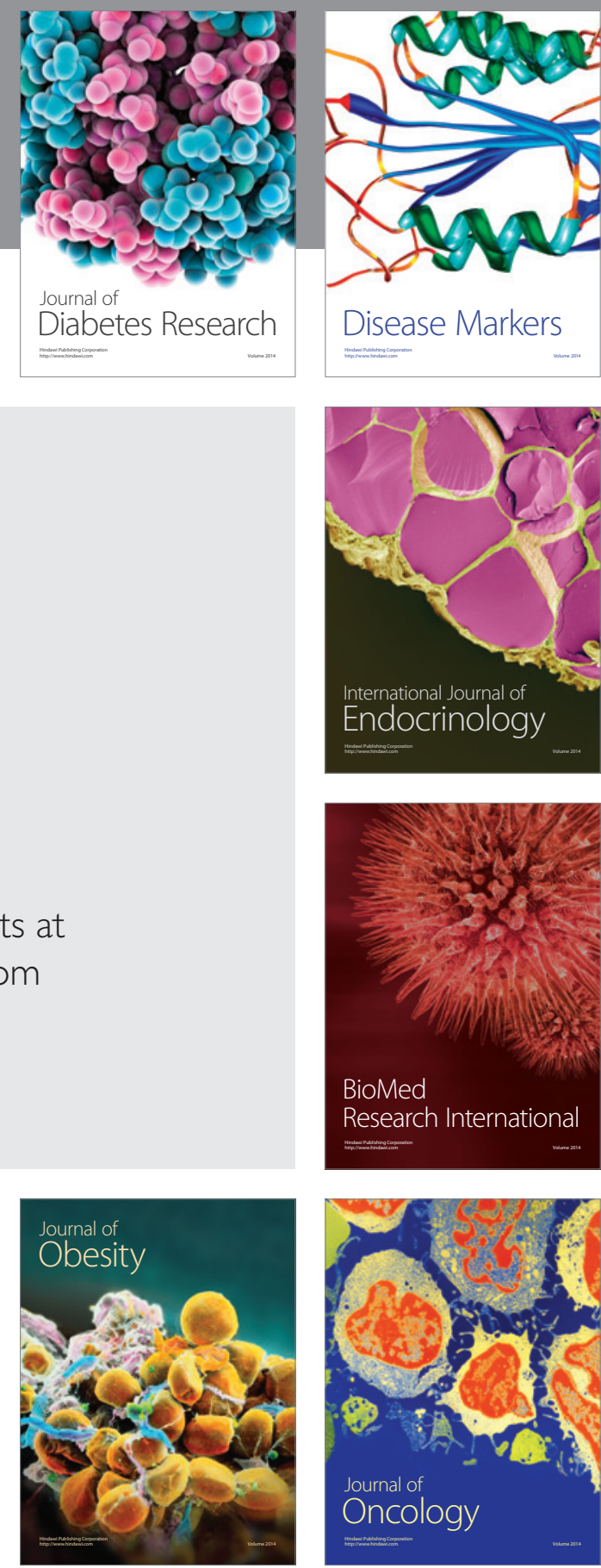

Disease Markers
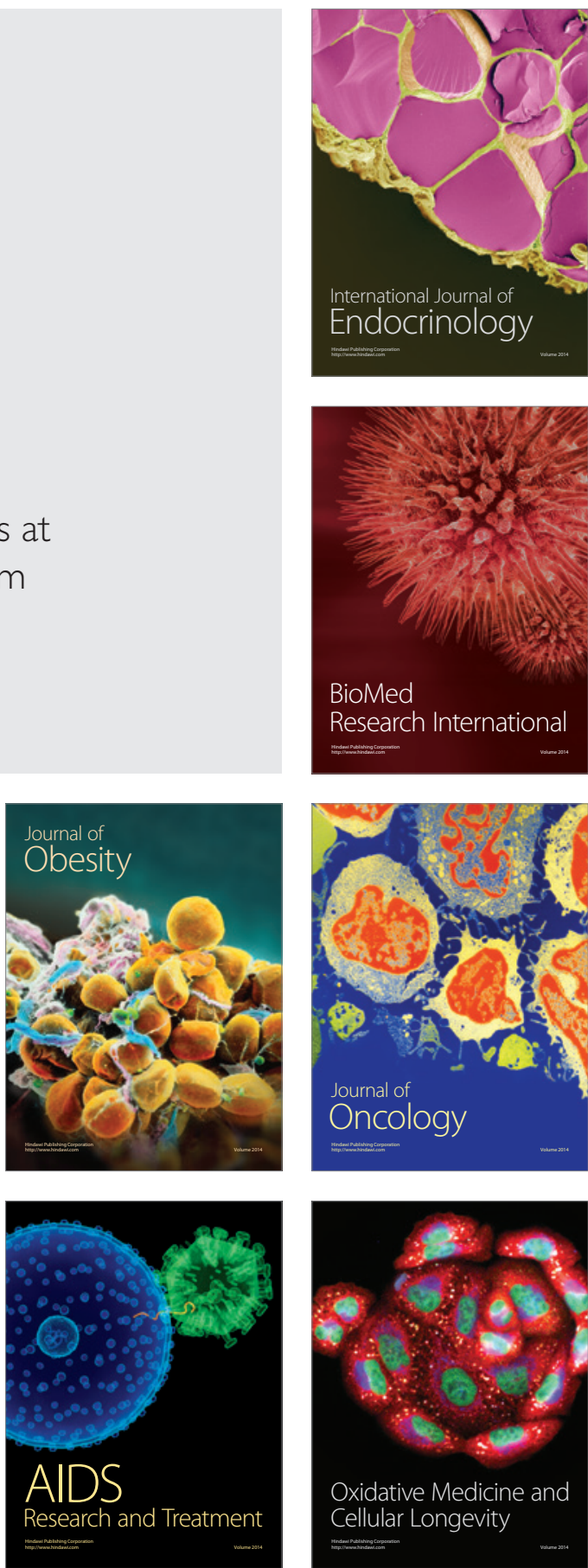